\title{
Solar source of energetic particles in interplanetary space during the 2006 December 13 event
}

\author{
C. $\mathrm{Li}^{1}$, Y. Dai ${ }^{2,3}$, J.-C. Vial ${ }^{2}$, C. J. Owen ${ }^{1}$, S. A. Matthews ${ }^{1}$, Y. H. Tang ${ }^{3}$, C. Fang ${ }^{3}$, and A. N. Fazakerley ${ }^{1}$ \\ 1 Mullard Space Science Laboratory, University College London, Dorking, Surrey RH5 6NT, UK \\ e-mail: cl2@mssl.ucl.ac.uk \\ 2 Institut d'Astrophysique Spatiale, Université Paris-sud 11 and CNRS, 91405 Orsay, France \\ 3 Department of Astronomy, Nanjing University, Nanjing 210093, PR China
}

Received 4 March 2009 / Accepted 12 June 2009

\begin{abstract}
An X3.4 solar flare and a fast halo coronal mass ejection (CME) occurred on 2006 December 13, accompanied by a high flux of energetic particles recorded both in near-Earth space and at ground level. Our purpose is to provide evidence of flare acceleration in a major solar energetic particle (SEP) event. We first present observations from ACE/EPAM, GOES, and the Apatity neutron monitor. It is found that the initial particle release time coincides with the flare emission and that the spectrum becomes softer and the anisotropy becomes weaker during particle injection, indicating that the acceleration source changes from a confined coronal site to a widespread interplanetary CME-driven shock. We then describe a comprehensive study of the associated flare active region. By use of imaging data from HINODE/SOT and SOHO/MDI magnetogram, we infer the flare magnetic reconnection rate in the form of the magnetic flux change rate. This correlates in time with the microwave emission, indicating a physical link between the flare magnetic reconnection and the acceleration of nonthermal particles. Combining radio spectrograph data from Huairou/NOAC, Culgoora/IPS, Learmonth/RSTN, and WAVES/WIND leads to a continuous and longlasting radio burst extending from a few GHz down to several $\mathrm{kHz}$. Based on the photospheric vector magnetogram from Huairou/NOAC and the nonlinear force free field (NFFF) reconstruction method, we derive the 3D magnetic field configuration shortly after the eruption. Furthermore, we also compute coronal field lines extending to a few solar radii using a potential-field source-surface (PFSS) model. Both the so-called type III- $l$ burst and the magnetic field configuration suggest that open-field lines extend from the flare active region into interplanetary space, allowing the accelerated and charged particles escape.
\end{abstract}

Key words. acceleration of particles - Sun: flares - Sun: coronal mass ejections (CMEs) - Sun: magnetic fields

\section{Introduction}

Based on decades of observations, it has been widely accepted that there are two classes of solar energetic particle (SEP) events (Cane et al. 1986; Kallenrode et al. 1992). The first class, lasting for hours, known as impulsive SEP events, are characterized by a distinct enhancement of high- $Z$ elemental abundance (Reames et al. 1994) that presumably arises from resonant waveparticle interaction in the flare reconnection region (Roth \& Temerin 1997; Miller 1997). The second class, lasting for days and known as gradual SEP events, are more intense and geoeffective. Such gradual events, so-called major events, are always associated with fast coronal mass ejections (CMEs) that drive shocks capable of accelerating the particles they encounter (Kahler et al. 1986; Cane et al. 1991). However, fast CMEs are associated with flares, representing different manifestations of the same magnetic energy release process (Harrison 1995; Zhang et al. 2001; Wang et al. 2003). Both are capable of accelerating particles that form part of an SEP event, so which process dominates the particle injection remains enigmatic.

The present paradigm decouples the source of the particles from flares in major events for two main reasons. First, the particle release time generally occurs somewhat later than the flare soft X-ray emission peak (Cliver et al. 1982; Kahler 1994). However, if we consider the effect of interplanetary scattering, and compare it to the flare nonthermal emissions (for instance hard X-ray, gamma-ray, and microwave emissions), the particle release time could still be consistent with the flare emission (Tang et al. 2006). Another argument against flare acceleration in major events is the belief that all flare-accelerated particles are trapped on closed magnetic field lines beneath the CME and cannot escape to interplanetary space (Reames 2002). However, Cane et al. (2002) found that major events are always accompanied by continuous and longlasting radio bursts from around $500 \mathrm{MHz}$ down to less than $1 \mathrm{MHz}$ generated by flareaccelerated electrons as they propagate away from the Sun. This indicates that there should be open-field lines extending from beneath CMEs such that interplanetary particles originating in flare regions might be expected in major events.

Observational results have been found to support the flare acceleration of SEPs in major events. The time-intensity profiles of the SEP events that occurred on 2000 July 14 and 2003 October 28 both displayed two injection peaks: an initial impulsive increase corresponding to the flare eruption, followed by a gradual component until the CME-driven shock arrived at Earth (Li et al. 2007a,b). It thus appears that there are two populations of SEPs: the prompt one displays an impulse-like increase with a high $\mathrm{Fe} / \mathrm{O}$ ratio, being flare-related; the delayed one has a slow intensity rise, and a low $\mathrm{Fe} / \mathrm{O}$ ratio, being $\mathrm{CME}$-driven shock related (Cane et al. 2003). Desai et al. (2004) examined five interplanetary shocks associated with SEP events, and found that the $\mathrm{Fe} / \mathrm{O}$ ratio increases with increasing energy. This observation contradicts the anticipation that the shock-accelerated $\mathrm{Fe} / \mathrm{O}$ ratio will decrease with increasing energy. Furthermore, 
Cane et al. (2007) found that the $\mathrm{Fe} / \mathrm{O}$ ratios in these events can decrease even when the shock is traveling through an Fe-rich ambient plasma. This observation does not support the proposal of shock acceleration of the suprathermal ion population, which is used to explain the high- $Z$ element abundance in major events (Tylka et al. 2005).

Numerical models also suggest that mixed particle acceleration by both flares and CME-driven shocks provide much better fits to the in-situ observations. Li \& Zank (2005) found that the proton intensity profile of the flare-shock-mixed case shows an initial rapid increase, owing to the flare acceleration, and followed by a plateau similar to that of a pure shock case. Furthermore, Verkhoglyadova et al. (2008) showed a good agreement between the mixed acceleration modeled spectra of heavy ions and the in-situ particle data.

So the classical two-type classification of SEP events appears not to be so clear-cut. In an event, both the flare and CMEdriven shock could accelerate particles. To determine their relative roles in particle acceleration during different phases of an event is the main goal of this work. Fortunately, the event that occurred on 2006 December 13, the last ground level enhancement (GLE) event of solar cycle 23, gives us such an excellent opportunity.

This paper is organized as follows: Sect. 2 starts with an overview of the instrumentations applied in this study. Section 3 presents the observational results in detail, including the dynamics of SEPs (time history, spectrum and anisotropy) and a study of the associated flare active region (magnetic reconnection rate and radio dynamic spectra). In Sect. 4, we use both the nonlinear force-free field (NFFF) reconstruction method and the potentialfield source-surface (PFSS) model to obtain the coronal magnetic configurations above the flare active region. In Sect. 5, we discuss interpretation of the above analysis. Section 6 is dedicated to conclusions.

\section{Instrumentations}

In this study, neutron monitor (NM) and spacecraft particle data are presented to help us interpret the SEP event, and multiwavelength observations are used to study the associated flare active region.

\subsection{Particle emission}

The proton data in the energy range of $15-500 \mathrm{MeV}$, with a temporal resolution of $1 \mathrm{~min}$, was obtained by Geostationary Operational Environment Satellite (GOES) 11, which is in geostationary orbit above the Pacific ocean. The one-minuteaveraged solar cosmic ray (SCR) intensity was obtained from the Apatity NM, which is located at N67.57E33.40 and has a very low cut-off rigidity of $0.63 \mathrm{GV}$. At the time of the event, the Apatity NM was viewing in the direction towards the Sun along the interplanetary magnetic field (IMF) lines, so it was in a suitable position to detect the direct prompt SCR intensity.

The electron data from the Electron, Proton, and Alpha Monitor (EPAM) onboard Advanced Composition Explorer (ACE) was used in the 53-103 keV and 42-65 keV energy band with, respectively, temporal resolutions of $12 \mathrm{~s}$ and 5-minaveraged. ACE orbits the L1 libration point which is a point of Earth-Sun gravitational equilibrium about 1.5 million $\mathrm{km}$ upstream from Earth. The ACE/EPAM is designed to make in-situ measurements of ions and electrons in interplanetary space. It is composed of five detectors with either 4 or 8 sectors that divide the spin space into approximately equal regions, give us the opportunity to investigate particles anisotropy. Details are given in Gold et al. (1998). Using the LEFS60 detector, which is one of the two Low-Energy Foil Spectrometer systems (LEFS60 and LEFS150) that points in a sunward direction, we obtain electron intensities in 8 angular sectors in the 42-65 keV energy band which allows us to determine the anisotropy of interplanetary particles.

\subsection{Multi-wavelength emission}

The light curve of the flare active region and the post flare looplike structure were obtained from the Transition Region and Coronal Explorer (TRACE, Handy et al. 1999) in the $195 \AA$ passband. The hard X-ray (HXR) flux in 12-25 keV energy band was obtained from the Reuven Ramaty High Energy Solar Spectroscopic Imager (RHESSI, Lin et al. 2002). Using the maximum entropy method (MEM-Sato algorithm, Sato et al. 1999), we also reconstructed the RHESSI HXR sources respectively in $12-25 \mathrm{keV}$ and $25-50 \mathrm{keV}$ energy band.

The imaging data obtained from Hinode Solar Optical Telescope (Hinode/SOT, Tsuneta et al. 2008) in the Ca II H line at $396.85 \mathrm{~nm}$ were used to investigate the evolution of the chromospheric flare ribbons, and the Michelson Doppler Imager (MDI, Scherrer et al. 1995) onboard the Solar and Heliospheric Observatory (SOHO) provided the longitudinal magnetic field map. To investigate the coronal large scale disturbance, the $195 \AA$ images of Extreme-Ultraviolet Imaging Telescope (EIT, Delaboudinière et al. 1995) on board the SOHO were also used.

The vector magnetogram, the microwave radio dynamic spectrum ranging from $2.6-3.8 \mathrm{GHz}$, and the radio flux at $3.8 \mathrm{GHz}$ were all obtained from the Huairou Station of the National Astronomical Observatories of China (Huairou/NAOC). The decimeter radio dynamic spectrum ranging from 180 to $1800 \mathrm{MHz}$ was obtained from Culgoora radio observatory, which is one of the stations of IPS radio and space services (IPS), it has a temporal resolution of $3 \mathrm{~s}$. The metric radio dynamic spectrum ranging from 25 to $180 \mathrm{MHz}$ was obtained from the solar radio spectrograph located at Learmonth in Australia, which is one of the instruments of the Radio Solar Telescope Network (RSTN). It has a temporal resolution of $3 \mathrm{~s}$. The decameter and hectometer $(\mathrm{DH})$ radio dynamic spectra in the frequency range of $20 \mathrm{kHz}-13.875 \mathrm{MHz}$ and with a temporal resolution of $1 \mathrm{~min}$, was recorded by the WAVES experiment (Bougeret et al. 1995) onboard the Wind spacecraft.

\section{Observations}

\subsection{Dynamics of SEPS}

On 2006 December 13, an X3.4 class flare occurred with a fast halo CME, bathing the Earth in a high flux of SEPs and a strong interplanetary shock. In this section, we present the dynamics of SEPs during the major event.

\subsubsection{Time history}

Figure 1 shows the SXR, HXR, and TRACE light curve histories (upper panel), together with the rise of electron, proton, and SCR count rates (lower panel). This figure indicates that the SXR event in GOES 1-8 $\AA$ started at 02:15 UT, peaked at 02:40 UT, followed by a $\sim 2 \mathrm{~h}$ decay phase. The HXR emission in 12-25 keV was observed by RHESSI from 02:29 UT, peaked at 02:32 UT, with a decay phase lasting to $03: 10$ UT. The derivative of the SXR profile peaked at 02:30 UT, which manifests as 


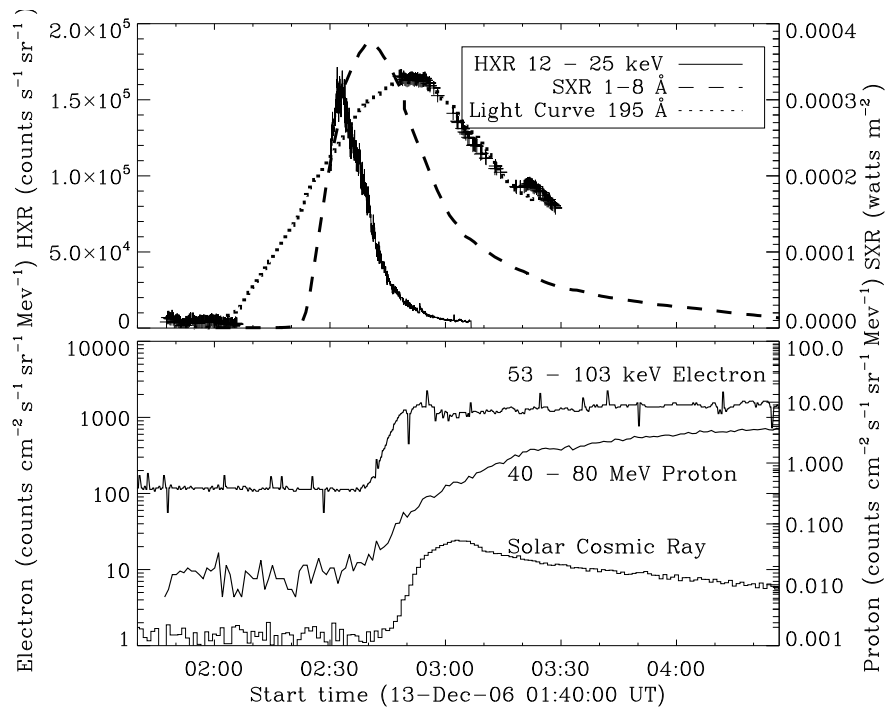

Fig. 1. Temporal profiles of electrons, protons, and SCRs, compared with SXR emission, HXR emission, and light curve of the flare active region. Upper panel: SXR flux (1-8 A, GOES), HXR flux (12-25 keV, RHESSI), and light curve of the active region (195 A, TRACE) in arbitrary unit, plus symbols indicate the data number (DN). Lower panel: electron intensity (53-103 keV, ACE/EPAM) with a temporal resolution of $12 \mathrm{~s}$, one-minute-averaged proton intensity (40-80 MeV, GOES), and SCRs counts rate (Apatity NM) in arbitrary unit.

an approximate match in time to the maximum of HXR emission. This consistent with expectations from the Neupert effect (Neupert 1968) which describes the relationship between the energy input by the nonthermal electrons and the plasma's thermal response. The light curve of the flare active region ( $X:$ [ 250 , 500], $Y$ : [-200, -50]) in unit of data number (DN, plus symbols in the upper panel) obtained from TRACE $195 \AA$ is also plotted. Although there was a data gap between 02:05 UT and 02:47 UT, we use a polynomial fit (short dashed line in the upper panel) to the DN and this predicts the peak time as 02:50 UT.

The arrival of energetic particles are traced by ACE/EPAM, GOES-11, and the Apatity NM (shown in the lower panel of Fig. 1). We assume that energetic particles travel along the IMF lines at a speed of $v$ with no scattering. Thus, with respect to the flare emission time, we estimate the particle release time by subtracting $\Delta t=S / v-8.3 \mathrm{~min}$ from the observed time at $1 \mathrm{AU}$, where $S$ is the length of IMF lines and $v$ is the velocity of energetic particles. $S \sim 1.1$ AU corresponding to a solar wind velocity of about $700 \mathrm{~km} \mathrm{~s}^{-1}$ during this event. The intensity onset is determined by $3 \sigma$ excess above background, where $\sigma$ is the standard deviation of the particle count rates. For GOES $\mathrm{P}_{5}$ (40-80 MeV) and ACE electrons (53-103 keV), we take $v$ to be approximately $0.4 c$ and $0.6 c$, where $c$ is the velocity of light. Then the evaluated proton and electron release times are respectively 02:45 UT and 02:41 UT. For Apatity NM SCRs, because of its low cut-off rigidity, we take the associated proton energy as the atmospheric absorption cut-off energy of $435 \mathrm{MeV}$ and $v$ to be $0.7 c$. Then the evaluated SCRs release time is 02:45 UT. Combining all the results and taking the systematic errors into account, we obtain the SEPs release time is 02:43 UT \pm 3 min.

From Fig. 1, the comparison between the increase profiles of SEPs and SXR emission, HXR emission, $195 \AA$ light curve of the flare active region, and the above analysis, it is found that the particle release time coincides with the flare emission, namely
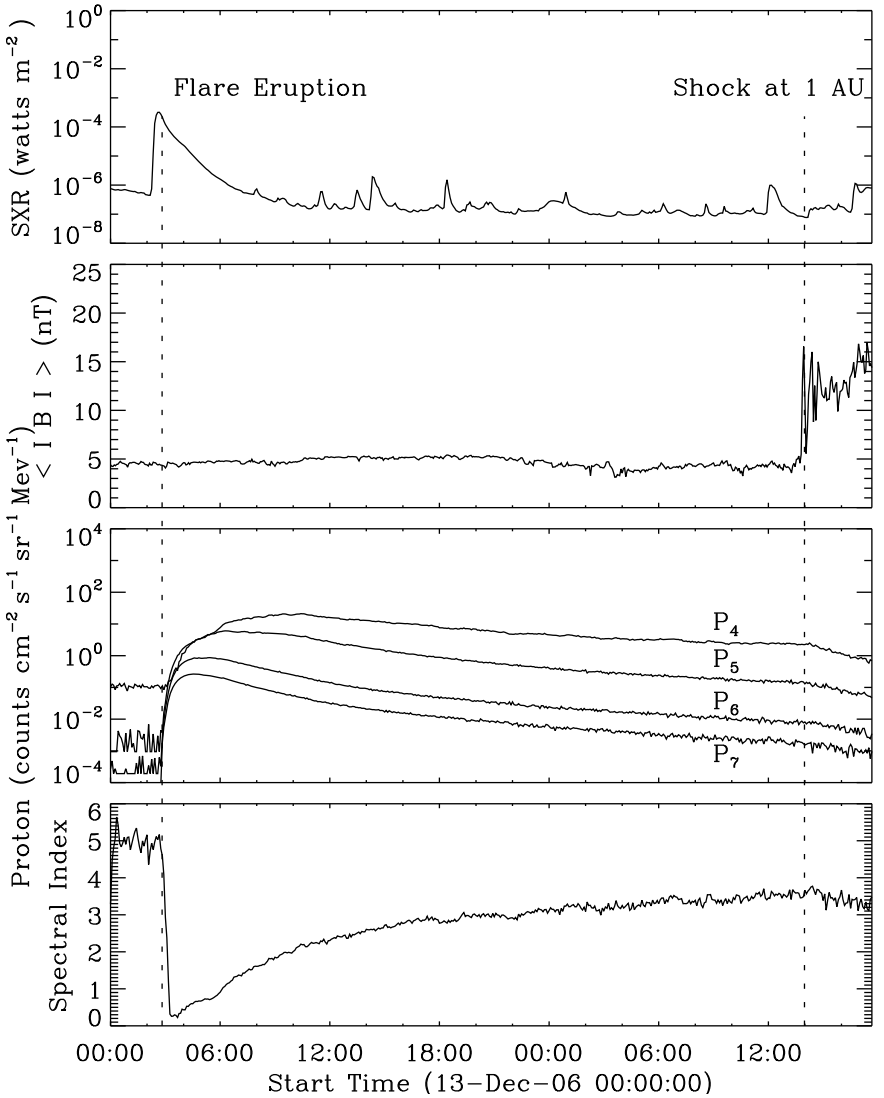

Fig. 2. From top to bottom: SXR flux (1-8 $\AA$, GOES), magnetic field magnitude (ACE/MAG), proton intensity (15-500 MeV, GOES), and spectral index evaluated during the injection of protons. Vertical dashed lines indicate the time of flare eruption and CME-driven shock reaches $1 \mathrm{AU}$.

with the rising phase of the $195 \AA$ light curve, the peak of the SXR emission, and the decay phase of the HXR emission.

\subsubsection{Spectrum}

Figure 2, from top panel to bottom, respectively shows temporal profiles of SXR flux, magnetic field magnitude, proton intensity, and derived proton spectral index for a period of two days. It is found that the injection profiles of protons have an impulsive component during the flare emission, which is followed by a gradual one. The latter lasts until the CME-driven shock reaches $1 \mathrm{AU}$ at 14:00 UT on 14 December, as marked by vertical dashed lines, when the magnetic field shows large increase, while the proton intensity shows an obvious decrease.

Assuming a power law spectrum $f(E) \propto E^{-\gamma}$, and using the different channel data from GOES in the energy range of 15$500 \mathrm{MeV}$, we get the proton spectral index during the particle injection, which is shown in the bottom panel of Fig. 2. It shows a soft background spectrum with an index $\gamma \sim 5$ before the SEP event. This suddenly becomes obviously hard during the flare eruption, then becomes increasely softer during the particle injection until the CME-driven shock reaches $1 \mathrm{AU}$.

Furthermore, we present the spectra at three individual times in Fig. 3. At the first peak flux, consistent with velocity dispersion that results in lower energetic particles reaching the peak a bit later than the higher ones, the spectral index $\gamma \sim 1.6$; at 18:00 UT 13 December, the spectral index $\gamma \sim 2.7$; at 14:00 UT 14 December, when the CME-driven shock reaches $1 \mathrm{AU}$, the 


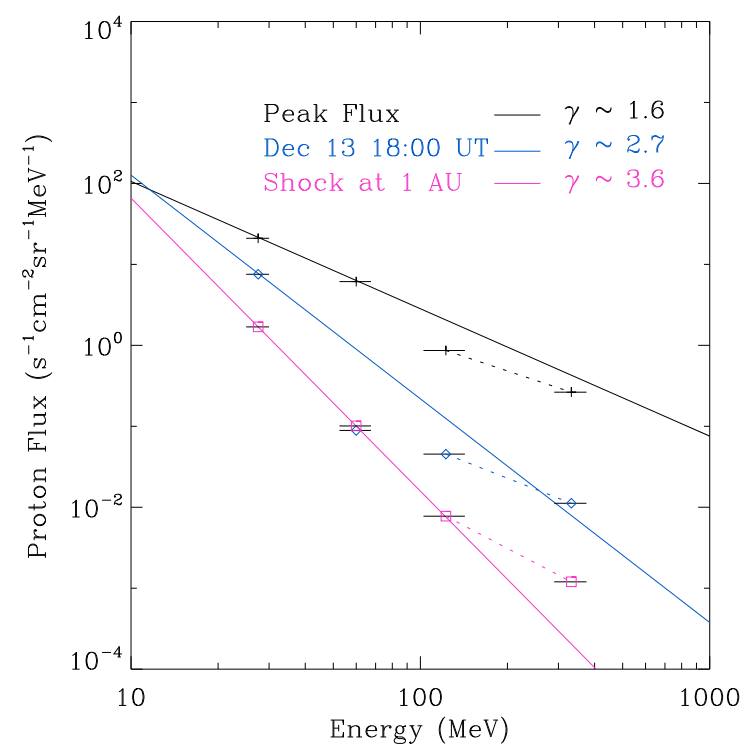

Fig. 3. The spectra at three different times during the SEP event. The data are obtained from GOES with the energy range of 15-500 MeV. Note that the spectrum becomes softer and softer during the proton injection. Dashed lines indicate spectral hardening in high energy band.

spectral index $\gamma \sim 3.6$. It is evident that the spectrum becomes softer and softer during particle injection. Another interesting phenomena to note is that the spectra at all three times appear to become harder in high energy band, which are shown by the dashed lines.

\subsubsection{Anisotropy}

In Fig. 4, three anisotropy pie plots derived from electron intensities of 8 sectors of ACE/EPAM LEFS60 detector are shown during the SEP event, each of them corresponds to the individual time of the spectrum shown in Fig. 3. The large anisotropy at 03:00 UT 13 December is clearly seen in the left panel of Fig. 4, where the electron count rates reach nearly $4000 \mathrm{c} \mathrm{s}^{-1}$ in sectors 3 and 4, smaller count rates are recorded by sectors 2 and 5 . The very weak anisotropy at 18:00 UT 13 December is shown in the middle panel, where only sector 8 records obviously smaller count rates than other sectors. From the above two panels, we can surmise the accelerated particles direction of travel relative to the spacecraft is from sectors 3 and 4 to sectors 7 and 8 during the first several hours. The right panel shows the nearly isotropic distribution at 14:00 UT 14 December, indicating particles impact the spacecraft from all the directions when the CME-driven shock reaches the near-Earth space. Such a change in anisotropy suggests that particle acceleration source shifts from a confined coronal site to a widespread interplanetary region.

\subsection{Associated flare active region}

The source region of the major event was rooted in NOAA active region 10930. When the flare occurred, the position of this region was roughly at S06W24, which was near to the nominal well-connected region or the foot-point of interplanetary magnetic field (IMF) lines connecting the Sun to the Earth. In this section, we present observations of the associated flare active region.

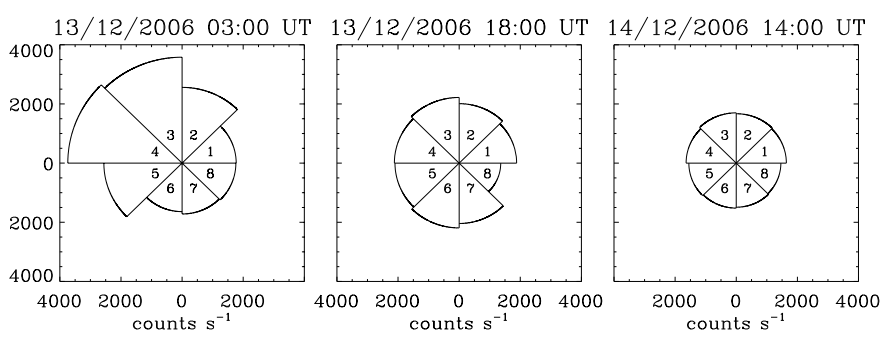

Fig. 4. Anisotropy plots from ACE/EPAM (42-65 keV electron). Left panel: strong anisotropy is seen in sectors 3 and 4 measured at 03:00 UT 13 December. Middle panel: weak anisotropy is only seen in sector 8 at 18:00 UT 13 December. Right panel: nearly isotropic distribution measured at 14:00 UT 14 December.

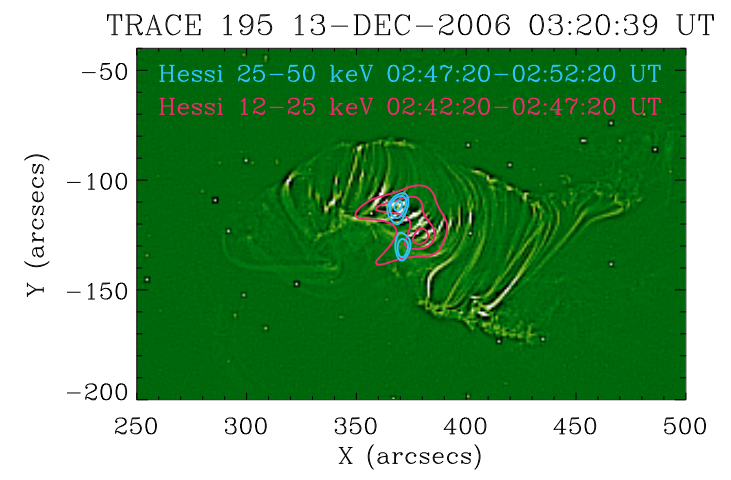

Fig. 5. Post flare loop-like structure shown by the high-pass filtered TRACE $195 \AA$ Amage at 03:20:39 UT, overlaid by RHESSI hard X-ray sources (Rotating modulation collimators $4-8$, with MEM-Sato image reconstruction algorithm). Blue contour lines indicate $25-50 \mathrm{keV}$ hard X-ray sources integrated from 02:47:20 to 02:52:20 UT, and red 12$25 \mathrm{keV}$ integrated from 02:42:20 to 02:47:20 UT.

\subsubsection{Flare magnetic reconnection}

Figure 5 shows the post-flare loop-like structure in the highpass filtered TRACE $195 \AA$ image at 03:20:39 UT, which is overlaid with MEM-Sato reconstructed RHESSI hard X-ray sources. Blue contour lines indicate the $25-50 \mathrm{keV}$ nonthermal bremsstrahlung sources integrated from 02:47:20 to 02:52:20 UT, and red contour lines indicate 12-25 keV thermal sources integrated from 02:42:20 to 02:47:20 UT. It is evident that the flare is a typical two-ribbon system.

From the analytical (Forbes \& Lin 2000) and numerical (Chen et al. 1999) results, along with a large amount of tworibbon flare observations, it is accepted that the flare ribbon expansion in the chromosphere is the signature of progressive magnetic reconnection in the corona. The free energy contained in the non-potential magnetic field can be rapidly dissipated into particle acceleration and plasma heating at the reconnection region. How fast the magnetic reconnection proceeds corresponds to how quickly the ribbons expand and how strong the magnetic field is that the ribbons sweep through. Thus we can estimate the magnetic reconnection rate in the form of the magnetic flux change rate (Qiu et al. 2002):

$\varphi_{\mathrm{rec}}=\frac{\partial \Phi_{B}}{\partial t}=\frac{\partial}{\partial t} \int B_{n} \mathrm{~d} A$,

where $\Phi_{B}$ is the magnetic flux, $B_{n}$ the magnetic field that the flare ribbons sweep through, and $\mathrm{d} A$ the new area swept through by the ribbons. It can be further compared with the microwave 


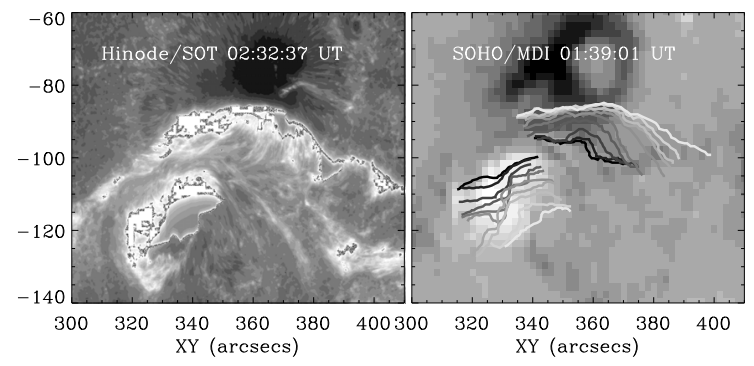

Fig. 6. Left panel: Snapshot of the X3.4 flare observed by Hinode/SOT at $396.85 \mathrm{~nm}$. Right panel: SOHO/MDI magnetogram of the NOAA active region 10930 with the trajectories of the two ribbons superposed. The color from dark to white indicates the separation process of the ribbons.

emission to understand the role of the flare magnetic reconnection in accelerating nonthermal particles.

Figure 6 shows the X3.4 flare observed by Hinode/SOT and the SOHO/MDI magnetogram of the flare active region with the trajectories of the two ribbons superposed. The Hinode/SOT Broadband Filter Imager (BFI) produces images with broad spectral resolution in 6 bands (CN band, Ca II H line, G-band, and 3 continuum bands) at the highest spatial resolution, $0.0541 \mathrm{arcsec} /$ pixel, over a $218^{\prime \prime} \times 109^{\prime \prime}$ field of view (FOV). The Ca II H line at $396.85 \mathrm{~nm}$ image shows the chromospheric structure, so we use it to evaluate the flare ribbon separation and get the new area $\mathrm{d} A$ swept through by the ribbons, the time cadence is $\sim 2$ min during the flare eruption. The magnetic field $B_{n}$ can be obtained from the longitudinal component of the MDI magnetogram at 01:39:01 UT before the flare eruption. Then the magnetic reconnection rate described in formula (1) can be obtained.

The rate of magnetic flux change is evaluated for each of the two ribbons, which respectively sweep through positive and negative magnetic field, as $\varphi_{\mathrm{rec}(+)}$ and $\varphi_{\mathrm{rec}(-)}, \varphi_{\mathrm{rec}}$ is the average of them. In Fig. 7, the inferred magnetic reconnection rate is shown in comparison with the microwave emission at $3.8 \mathrm{GHz}$ generated by high-speed electrons via synchrotron. Both the radio flux of right-handed circular polarization (RHCP) and left-handed circular polarization (LHCP) are shown. Generally speaking, a good temporal correlation is found. Furthermore, the correlation coefficient of $\varphi_{\mathrm{rec}(+)}, \varphi_{\mathrm{rec}(-)}$, and $\varphi_{\text {rec }}$ with the RHCP radio flux is calculated as $0.72,0.80$, and 0.87 , with the LHCP radio flux as $0.84,0.74$, and 0.90 . Such a good correlation indicates a strong physical link between the flare magnetic reconnection and the energy release, and the flare magnetic reconnection plays an important role in accelerating nonthermal particles.

\subsubsection{Radio dynamic spectra}

Solar radio bursts provide a clue to examine whether or not particles accelerated in the flare site could escape and be observed in near-Earth space. We combine various spectrographs to analyze the radio dynamic spectra over an extended frequency range. Figure 8 shows a continuous and longlasting type III radio burst from $\sim 1 \mathrm{GHz}$ to $20 \mathrm{kHz}$, that starts at 02:24 UT and lasts about $20 \mathrm{~min}$ in low frequency. This kind of type III burst, or so-called type III- $l$ burst, shows characteristics of low-frequency extension and longlasting duration (longer than the usual type III burst of 5-10 min). A type II radio burst starts at 02:27 UT with an initial frequency of $180 \mathrm{MHz}$ is also recorded.

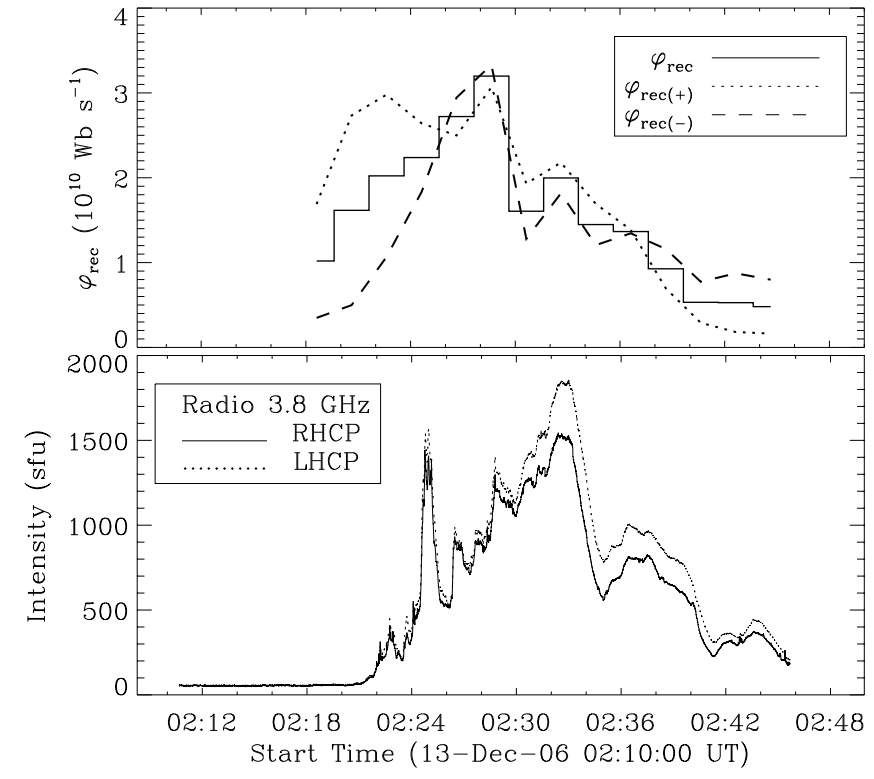

Fig. 7. Magnetic reconnection rate inferred from the X3.4 two-ribbon flare compared with the microwave radio emissions at $3.8 \mathrm{GHz}$. In the upper pannel, the magnetic reconnection rate is evaluated for each of the two ribbons as $\varphi_{\mathrm{rec}(+)}$ and $\varphi_{\mathrm{rec}(-)}, \varphi_{\mathrm{rec}}$ is the mean value of the two. In the lower panel, the solid line is the radio flux of right-handed circular polarization (RHCP), dashed line of Left-handed (LHCP).

Radio bursts (type I through V) are generated by electron streams as they propagate along magnetic field lines from solar corona to interplanetary medium (Wild et al. 1963). The emission frequency corresponds to the local plasma frequency $\left(9 \sqrt{n_{\mathrm{e}}} \mathrm{kHz}\right)$. In detail, the plasma frequency of $1 \mathrm{GHz}$ corresponds to $n_{\mathrm{e}} \sim 10^{10} \mathrm{~cm}^{-3}$ and a coronal height of less than $0.1 R_{\mathrm{S}}$ above the photosphere, $20 \mathrm{kHz}$ corresponds to $n_{\mathrm{e}} \sim 10 \mathrm{~cm}^{-3}$ and the near-Earth plasma. Thus the type III- $l$ burst suggests the existence of open magnetic field lines from a low coronal site, probably the flare active region, to the interplanetary space.

An phenomena to note in Fig. 8 is that the type III- $l$ burst seems to be an extension of the $2.6-3.8 \mathrm{GHz}$ microwave emission. This suggests that the related electrons arise from the same flare magnetic reconnection region, one population is trapped in closed magnetic field and generates microwave emission through synchrotron, another population escapes along opened magnetic field and generates type III- $l$ burst through plasma emission.

\section{Magnetic field modeling}

Another way to answer the question consists of examining the magnetic field configuration above the flare active region. There is currently no direct high-quality measurement of coronal magnetic field. One method of obtaining the magnetic field configuration is the precise extrapolation of the accurately observed photospheric magnetic field. Song et al. (2006, 2007) developed a fast upward integration NFFF reconstruction method. Instead of using finite difference to express the basic NFFF partial derivatives, they introduced smooth continuous functions to approach magnetic field values, and a self-consistent compatibility condition for field boundary values was considered.

Based on the photospheric vector magnetogram from Huairou/NAOC at 03:07 UT, just after the flare eruption, and the NFFF reconstruction method, the 3D magnetic field 


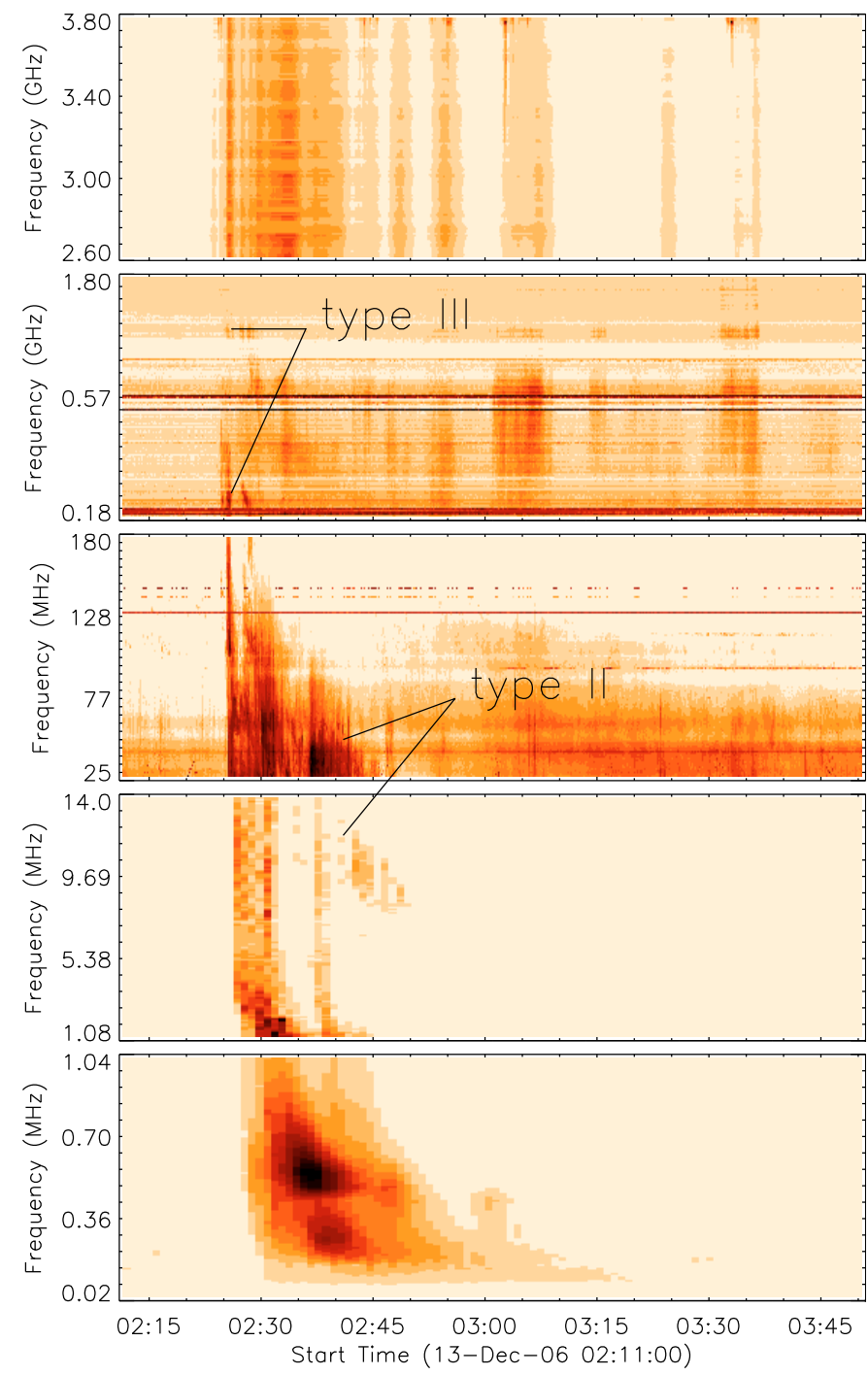

Fig. 8. From top to bottom: the radio dynamic spectra combining Huairou/NAOC with the frequency range of $2.6-3.8 \mathrm{GHz}$, Culgoora/IPS of 180 to $1800 \mathrm{MHz}$, Learmonth/RSTN of 25-180 MHz, WAVES/WIND Rad2 1.075 MHz-13.875 MHz, and Rad1 of $20 \mathrm{kHz}-$ $1.04 \mathrm{MHz}$.

configuration above the flare active region is obtained and shown in Fig. 9. The magnetogram has magnetic field noise levels of $\pm 10 \mathrm{G}$ for longitudinal fields and $\pm 100 \mathrm{G}$ for transversal fields, the spatial resolution is $2.8^{\prime \prime}$, and the spatial domain is $169^{\prime \prime} \times 169^{\prime \prime}$. The magnetic field configuration is computed upwards to a height of $122000 \mathrm{~km}, 0.17 R_{\mathrm{s}}$ above the photosphere. It is evident that the magnetic configuration shows non-potential complexity and loop-like structure in the low corona, gradually becoming much potential and then "open" in the high corona above $\sim 0.1 R_{\mathrm{s}}$. Similar extrapolation results by various reconstruction methods are also derived by Schrijver et al. (2008) and Guo et al. (2008). Note that the "open" lines mainly extend along the northwest ( $X Y$ direction) of the solar disk, in this direction charged particles can easily access the nominal well-connected region and then be released into interplanetary space.

Furthermore, we use the PFSS model developed by Schrijver \& DeRosa (2003), which is available in the IDL-based solar software (SSW) package, to identify the large-scale coronal magnetic configuration. This model assumes a spherical source surface (where the magnetic energy density equals to the plasma

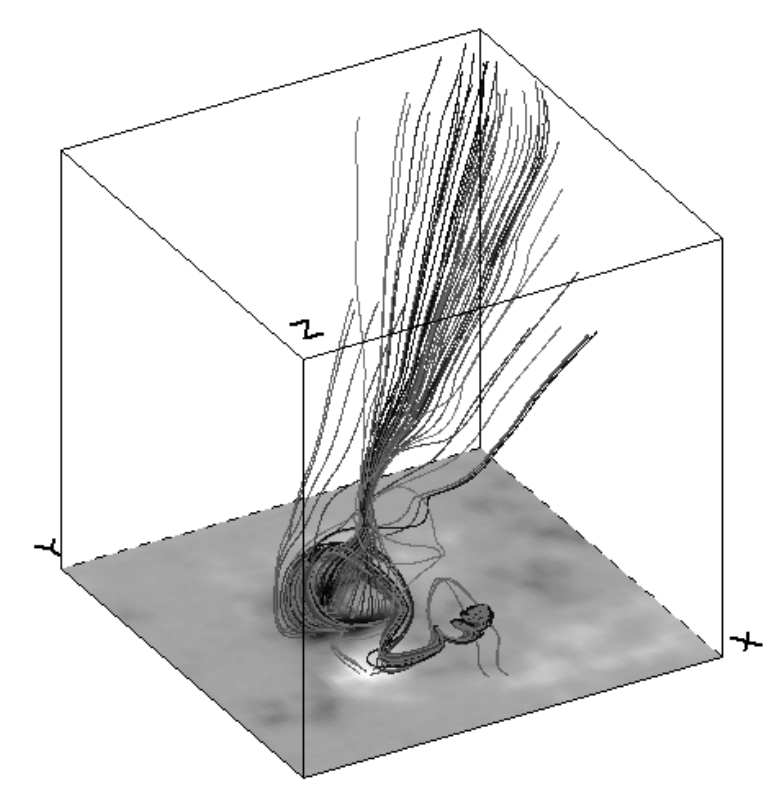

Fig. 9. Magnetic field configuration of the NFFF lines computed based on the photospheric vector magnetogram of Huairou/NOAC. The spatial domain of the magnetogram is $169^{\prime \prime} \times 169^{\prime \prime}$, and the calculated height is $122000 \mathrm{~km} . X$ and $Y$ axes respectively indicate west and north direction of the solar disk, and $Z$ indicates radial direction.

energy density). Inside of this surface the magnetic field is derived from a potential Laplace equation: $\nabla^{2} \phi$, outside which the magnetic field is frozen into the solar wind (Schatten et al. 1969). It has been successfully applied to confirm the existence of coronal magnetic flux tubes, in which the type III radio sources originate (Klein et al. 2008).

Based on the photospheric longitudinal magnetogram from SOHO/MDI at 00:04 UT and the PFSS code, the coronal magnetic field lines extending to $2.5 R_{\mathrm{s}}$ are obtained and shown in Fig. 10. The green lines mark the closed-field lines, and red lines mark the open-field lines. The upper panel displays the view from the Earth, with the top of the map indicating solar north and the right, solar west. The lower panel displays the view from the solar north pole, map bottom towards to the Earth. As in the NFFF model the open-field lines, which are rooted in the active region, follow the westward direction to the well-connection region and extend to interplanetary space. Such open-field lines are a possible route for transporting particles from the flare site.

\section{Discussion}

\subsection{Interpretation of particle release times}

In Sect. 3.1.1, we find that there is no contradiction between the particle injection and the flare emission. However, the evaluated proton release time is $\sim 02: 45 \mathrm{UT}$, which is a few minutes later than the electron release at $\sim 02: 41$ UT. Similar delays are also reported by Klein et al. (2005) and Le et al. (2006) in the SEP events occurred on 1999 June 29, 2000 May 01, and 2005 January 20.

One may suppose that the electron release coincides with the flare eruption and is related to type III burst (Lin 1985), while protons are accelerated in the subsequent CME-driven shock and related to type II burst (Reames 1999). However, according to the observations from Culgoora/IPS, the type II burst started at 

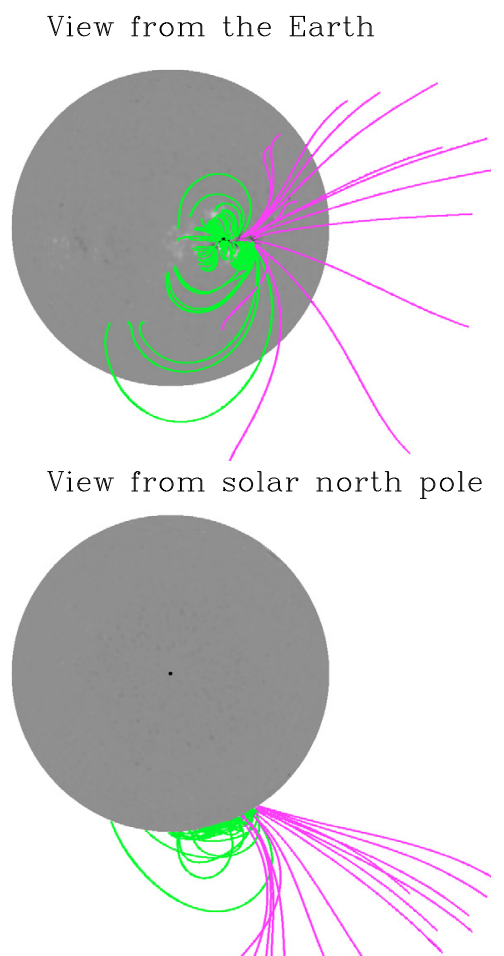

Fig. 10. Magnetic field configuration of the PFSS lines computed based on the photospheric longitudinal magnetogram of SOHO/MDI. The green lines mark the closed-field lines, and red lines mark the openfield lines. Upper panel: the view direction is from the Earth. Lower panel: the view direction is from the solar north pole.

02:27 UT, which is much earlier than both of the proton and electron releases. Shock acceleration has no species selectivity, so the question is why did it not accelerate electrons and protons at the same time? Another suggestion, introduced by Krucker \& Lin (2000), is that protons are released at a much higher altitude than electrons, and protons with successively lower energies are released as the CME-driven shock propagates to successively greater heights, corresponding to the release times being successively delayed. However, from analysis of the radio observations and the escaping deka-MeV protons, Klein \& Posner (2005) found that the initial particles are accelerated behind the front of the CME-driven shock. Moreover, it still cannot explain the same release times between relativistic SCRs and lower energetic protons in this event.

A reasonable possibility is that the interplanetary trajectories of protons and electrons are different due to their distinct pitchangle scattering and mean free paths. For instance, Krucker \& Lin (2000) analyzed 26 events, and found that the derived electron travel length is $1.1-1.3 \mathrm{AU}$, but the proton travel length is around 2 AU in 9 events. Using numerical models of interplanetary transport to fit the in-situ observations from the 2000 July 14 event, Bieber et al. (2002) found that the electron and proton mean free path are respectively $0.75 \mathrm{AU}$ and $0.27 \mathrm{AU}$. Thus, taking the interplanetary scattering and the mean free path of 0.08-0.3 AU (Palmer 1982) into account, the proton release time could be brought forward by a few minutes (Kahler 1994).

\subsection{Interpretation of particle spectra}

In Sect. 3.1.2, we derive the proton spectra and find significant change of the spectral index. For acceleration at a parallel shock,

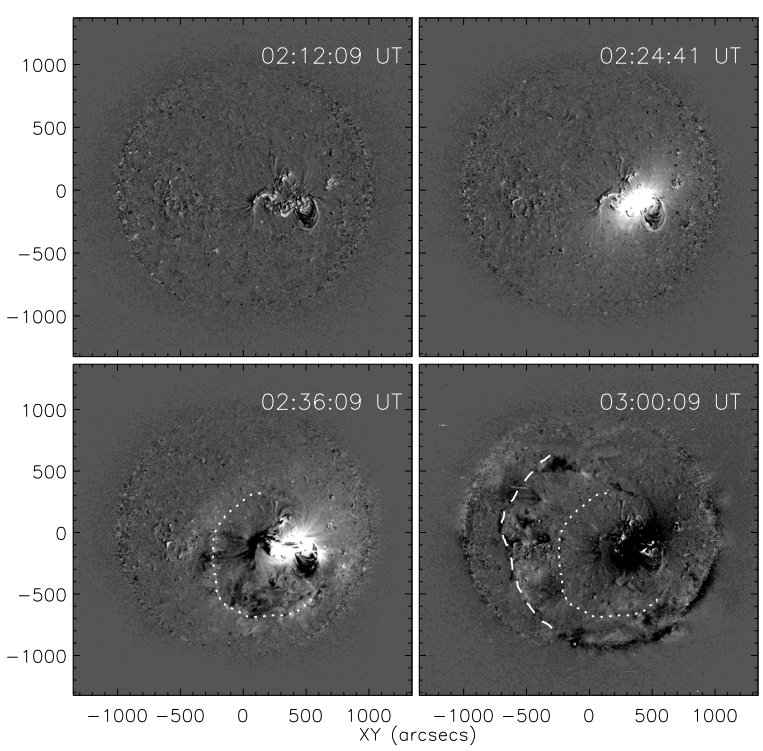

Fig. 11. The coronal disturbance on 2006 December 13. The four images show SOHO/EIT $195 \AA$ A ones at 02:12, 02:24, 02:36, and 03:00 UT with a pre-event image subtracted from them, respectively. Dashed lines indicate the fronts of the disturbance or the EIT wave.

particles gain energy by scattering freely between the converging upstream and downstream plasma without influencing the shock structure. The accelerated particle distribution function is a power law form in momentum (Blandford \& Ostriker 1978), $f(p) \mathrm{d}^{3} p p^{-\sigma} \mathrm{d}^{3} p$, where $f(p) \mathrm{d}^{3} p$ is the number of particles in the volume $\mathrm{d}^{3} p$ of momentum space per unit volume. The spectral index $\gamma$ depends only on $r$, inverse of the compression ratio, $\gamma=3 r /(r-1)$, where $r=u_{1} / u_{2}$ and $u_{1}\left(u_{2}\right)$ is the upstream (downstream) bulk plasma flow velocity. $r$ can be determined from the Rankine-Hugonoit conservation relation. With assumptions that the shock is planar (the gyro-radii of all particles are small compared to the curvature of the shock), the gas is ideal, and the adiabatic index $5 / 3$, the relation can be reduced to (Ferraro \& Plumpton 1966; Ellison \& Ramaty 1985):

$Q_{1} r^{2}+\left(5 Q_{1}+\frac{5}{3} M_{1}^{2}\right) r-\frac{20}{3} M_{1}^{2}=0$

where $Q_{1}$ is the upstream magnetic to gas pressure ratio and $M_{1}$ is the upstream Mach number. For the coronal shock, taking typical values of $T \sim 10^{6} \mathrm{~K}, n \sim 10^{9} \mathrm{~cm}^{-3}, B \sim 10 \mathrm{G}$, and the CME speed of $1774 \mathrm{~km} \mathrm{~s}^{-1}$, we get $r \doteq 2.7$. For the interplanetary shock at $1 \mathrm{AU}$, taking observational values from ACE data of $T \sim 10^{5} \mathrm{~K}, n \sim 10 \mathrm{~cm}^{-3}, B \sim 5 \mathrm{nT}$, and $M_{1} \sim 8$, we get $r \doteq 2.6$.

If particles are only accelerated by the CME-driven shock, we would expect almost the same spectral index between the initial coronal shock accelerated particles and the near-Earth interplanetary shock accelerated particles. However, it is not the case, and the fact is that the spectrum obviously becomes softer. In the current work, we are not able to exclude the influence of energetic storm particles (ESPs) during the passage of IP shocks, which are either accelerated at the front shocks or trapped in the vicinity of shocks. Although there are evidence that only ESPs with a few MeV or below show obvious enhancement in this event (Liu et al. 2008), and that only $40 \%$ of 330 shock-related ESP events with energies greater than $1.5 \mathrm{MeV}$ (Huttunen-Heikinmaa \& Valtonen 2009). 
We cannot exclude an ideal model in which the acceleration shifts from the coronal perpendicular shock to the interplanetary parallel shock. The coronal transient wave or EIT wave might provide the observational evidence. Krucker et al. (1999) found that the electron release appeared related to the passage of EIT waves. Torsti et al. (1999) found that the initial injection of deka-MeV protons occurred during the period when the EIT wave was traversing the western hemisphere of the Sun, which was interpreted as a moving skirt of a quasi-perpendicular shock wave. However, recent studies (Dalannée 2000; Harra \& Sterling 2003; Chen et al. 2005; Attrill et al. 2008; Dai et al. 2009) revealed that EIT waves are not real material shocks, but coronal manifestations of the expanding and opening magnetic fields. A large-scale coronal disturbance or EIT wave was observed during this event. From the running difference of EIT $195 \AA$ images (shown in Fig. 11), it is found that a large amount of coronal material was ejected around 02:36 UT or before, corresponding to the process from the coronal brightening to dimming. This process may open or reconfigure quite much of the magnetic field in the corona, allowing the flare-accelerated particles to escape into interplanetary space.

We find that the proton spectra appear to become harder in high energy band (shown by the dashed lines in Fig. 3). This is in contradiction with the pure shock acceleration which would produce a softer spectrum beyond $30 \mathrm{MeV}$ (Ellison \& Ramaty 1985), on the other hand, is in favor to the contribution of flare acceleration which is believed more prolific than the shock at producing high energy particles (Tylka et al. 2005).

The unusual features of spectra, including spectral softening during particle injection and spectral hardening in the high energy band, give a reasonable possibility of the mixed particle acceleration by both flare and the CME-driven shock.

\subsection{Interpretation of flare magnetic reconnection}

In Sect. 4.1.1, we derive the magnetic reconnection rate in the form of magnetic flux change rate. There still exists other forms, such as continuous blue shift (Schmieder et al. 1987) and separation velocity (Fletcher et al. 2004) of the flare ribbons. Both are consistent with the concept in this study that the observed signatures in chromosphere and the energy release in flares are due to the magnetic reconnection at coronal site.

Assuming the separation of flare ribbons is translational symmetry, and in a two-dimensional configuration, we can estimate the induced electric field $E_{\text {rec }}$ along the current sheet in the magnetic reconnection region by $E_{\mathrm{rec}}=V_{\|} B_{n}$ (deduced from formula 1 ), where $V_{\|}$is the horizontal velocity of the ribbon expansion, $B_{n}$ the longitudinal magnetic field that the flare ribbons sweep through. Given the magnetic field $650 \mathrm{G}$ in the flare active region, and the expansion velocity $14 \mathrm{~km} \mathrm{~s}^{-1}$ at the peak of $\varphi_{\mathrm{rec}}$, we get a maximum electric field of $\sim 10 \mathrm{~V} / \mathrm{cm}$.

Based on the DC electric field acceleration mechanism (Litvinenko \& Somov 1995), this value is effectively enough to accelerate protons to a GLE typical energy of $\sim \mathrm{GeV}$ in a time of $<0.1 \mathrm{~s}$. In this event, according to the registration of SCRs by middle and low latitude neutron monitors, the maximum energy of the protons is estimated to be $\sim 10 \mathrm{GeV}$ (Karapetyan 2008). For the electrons, based on the neutral beam current sheet theory (Martens 1988), the energy is always a factor $m_{\mathrm{e}} / m_{\mathrm{p}}$ less than proton energy, and could reach the value of $\sim \mathrm{MeV}$. However, the DC electric field acceleration mechanism could hardly produce the high- $Z$ elemental abundance, which probably arises from the stochastic acceleration or the resonant wave-particle interaction.

\section{Conclusions}

This study contributes to the ongoing debate about which process, flare or/and CME-driven shock, is responsible for particle injection in major events (Tylka \& Lee 2006; Cane et al. 2007; Gopalswamy 2008). By use of particle data from nearEarth spacecraft and ground-based neutron monitor, along with multi-wavelength observations of the flare active region, we analyze the roles of flare magnetic reconnection and CME-driven shock in accelerating SEPs during the 2006 December 13 event. The key conclusions are as follows:

1. The particle initial release time coincides with the flare emission. The spectrum becomes softer and the anisotropy becomes weaker during the particle injection, indicating the acceleration source may change from a coronal site in the flare active region to a widespread interplanetary CME-driven shock.

2. The inferred magnetic reconnection rate is temporally wellcorrelated with the evolution of the microwave emission, indicating a strong physical link between the flare magnetic reconnection and the acceleration of nonthermal particles.

3. The continuous longlasting type III- $l$ radio burst and the computed magnetic field configuration suggest the existence of open magnetic field lines extending from the flare active region to interplanetary space. Flare accelerated particles should thus be present in the major event.

Acknowledgements. It is a pleasure to thank M. T. Song for the use of his NFFF reconstruction method, and C. M. Tan, Y. Y. Deng for their help with the Huairou/NAOC radio data. We are very grateful to SOHO, TRACE, RHESSI, GOES, ACE, WIND, RSTN, IPS, and Apatity NM for their open-policy data. Hinode is a Japanese mission developed and launched by ISAS/JAXA, with NAOJ as domestic partner and NASA and STFC (UK) as international partners. C. Li acknowledge the financial assistance from Science \& Technology Facilities Council (STFC) of the UK.

\section{References}

Attrill, G., Harra, L. K., Van Driel-Gesztelyi, L., \& Démoulin, P. 2008, ApJ, L101

Bieber, J. W., Dröge, W., Evenson, P. A., et al. 2002, ApJ, 567, 622

Blandford, R. D., \& Ostriker, J. P. 1978, ApJ, 267, L433

Bougeret, J.-L., Kaiser, M. L., Kellogg, P. J., et al. 1995, Space Sci. Rev., 71, 5 Cane, H. V., McGuire, R. E., \& von Rosenvinge, T. T. 1986, ApJ, 301, 448 Cane, H. V., Reames, D. V., \& von Rosenvinge, T. T. 1991, ApJ, 373, 675

Cane, H. V., Erickson, W. C., \& Prestage, N. P. 2002, J. Geophys. Res., 107, 1315

Cane, H. V., von Rosenvinge, T. T., Cohen, C. M. S., \& Mewaldt, R. A. 2003, Geophys. Res. Lett., 30, 8017

Cane, H. V., Richardson, I. G., \& von Rosenvinge, T. T. 2007, Space Sci. Rev., 130,301

Cliver, E. W., Kahler, S. W., Shea, M. A., \& Smart, D. F. 1982, ApJ, 260, 362

Cliver, E. W., Kahler, S. W., \& Reames, E. V. 2004, ApJ, 605, 902

Chen, P. F., Fang, C., Ding, M. D., \& Tang, Y. H. 1999, ApJ, 520, 853

Chen, P. F., Fang, C., \& Shibata, K. 2005, ApJ, 622, 1202

Dai, Y., Auchère, F., Vial, J.-C., Tang, Y. H., \& Zong, W. G. 2009, ApJ, submitted Delaboudinière, J.-P., Artzner, G. E., Brunaud, J., et al. 1995, Sol. Phys., 162, 291

Delannée, C. 2000, ApJ, 545, 512

Desai, M. I., Mason, G. M., Wiedenbeck, M. E., et al. 2004, ApJ, 611, 1156 Ellison, D. C., \& Ramaty, R. 1985, ApJ, 298, 400

Ferraro, V. C. A., \& Plumpton, C. 1966, An Intruduction to Magneto-Fluid Mechanics, 2nd edn. (Oxford: Clarendon), 101

Fletcher, L., Pollock, J. A., \& Potts, H. E. 2004, Sol. Phys., 222, 279

Forbes, T. G., \& Lin, J. 2000, J. Atmos. Sol-Terr. Phys., 62, 1499

Gold, R. E., Krimigis, S. M., Hawkins, S. E., et al. 1998, Space Sci. Rev., 86, 541

Gopalswamy, N. 2008, 7th Annual International Astrophysics Conference, AIP Conf. Proc., 1039, 196

Gosling, J. T. 1993, J. Geophs. Res., 98, 18937

Guo, Y., Ding, M. D., Wiegelmann, T., \& Li, H. 2008, ApJ, 679, 1629 
Handy, B. N., Acton, L. W., Kankelborg, C. C., et al. 1999, Sol. Phys., 187, 229 Harra, L. K., \& Sterling, A. C. 2003, ApJ, 587, 429

Harrison, R. A. 1995, A\&A, 304, 585

Huttunen-Heikinmaa, K., \& Valtonen, E. 2009, Ann. Geophys., 27, 767

Kahler, S. W. 1994, ApJ, 428, 837

Kahler, S. W., Cliver, E. W., Cane, H. V., et al. 1986, ApJ, 302, 504

Kallenrode, S. W., Cliver, E. W., \& Wibberenz, G. 1992, ApJ, 391, 370

Karapetyan, G. G. 2008, Astropart. Phys., 30, 234

Klein, K.-L., \& Posner, A. 2005, A\&A, 438, 1029

Klein, K.-L., Krucker, S., Trottet, G., \& Hoang, S. 2005, A\&A, 431, 1047

Klein, K.-L., Krucker, S., Lointier, G., \& Kerdraon, A. 2008, A\&A, 486, 589

Krucker, S., \& Lin, R. P. 2000, ApJ, 542, L61

Krucker, S., Larson, D. E., Lin, R. P., \& Thompson, B. J. 1999, ApJ, 519, 864

Le, G. M., Tang, Y. H., \& Han, Y. B. 2006, Chin. J. Astron. Astrophys., 6, 751

Li, G., \& Zank, G. P. 2005, Geophys. Res. Lett., 32, 2101

Li, C., Tang, Y. H., Dai, Y., Zong, W. G., \& Fang, C. 2007a, A\&A, 461, 1115

Li, C., Tang, Y. H., Dai, Y., Fang, C., \& Vial, J.-C. 2007b, A\&A, 472, 283

Lin, R. P. 1985, Sol. Phys., 100, 537

Lin, R. P., Dennis, B. R., Hurford, G. J., et al. 2002, Sol. Phys., 210, 3

Litvinenko, Y. E., \& Somov, B. V. 1995, Sol. Phys., 158, 317

Liu, Y., Luhmann, J. G., Müller-Mellin, R., et al. 2008, ApJ, 689, 563

Miller, J. A. 1997, ApJ, 491, 939

Neupert, W. M. 1968, ApJ, 153, L59

Palmer, I. D. 1982, Rev. Geophys. Space Phys., 20, 335

Qiu, J., Lee, J., Gary, D. E., \& Wang, H. M. 2002, ApJ, 565, 1335

Reames, D. V. 1999, Space Sci. Rev., 90, 413
Reames, D. V. 2002, ApJ, 571, 63

Reames, D. V., Meyer, J. P., \& von Rosenvinge, T. T. 1994, ApJS, 90, 649

Roth, I., \& Temerin, M. 1997, ApJ, 477, 940

Sato, J., Kosugi, T., \& Makishima, K. 1999, PASJ, 51, 127

Schatten, K. H., Wilcox, J. M., \& Ness, N. F. 1969, Sol. Phys., 6, 442

Scherrer, P. H., Bogart, R. S., Bush, R. I., et al. 1995, Sol. Phys., 162, 129

Schmieder, B., Forbes, T. G., Malherbe, J. M., \& Machado, M. E. 1987, ApJ, 317, 956

Schrijver, C. J., \& DeRosa, M. L. 2003, Sol. Phys., 212, 165

Schrijver, C. J., DeRosa, M. L., Metcalf, T., et al. 2008, ApJ, 675, 1637

Song, M. T., Fang, C., Tang, Y. H., Wu, S. T., \& Zhang, Y. A. 2006, ApJ, 649, 1084

Song, M. T., Fang, C., Zhang, H. Q., et al. 2007, ApJ, 666, 491

Tang, Y. H., Li, C., \& Dai, Y. 2006, Proc. IAU Symp., 233, 417

Torsti, J., Kocharov, L. G., Teittinen, M., \& Thompson, B. J. 1999, ApJ, 510, 460

Tsuneta, S., Suematsu, Y., Ichimoto, K., et al. 2008, Sol. Phys., 249, 167

Tylka, A. J., \& Lee, M. A. 2006, ApJ, 646, 1319

Tylka, A. J., Cohen, C. M. S., Dietrich, W. F., et al. 2005, ApJ, 625, 474

Verkhoglyadova, O. P., Li, G., Zank, G. P., \& Hu, Q. 2008, 7th Annual International Astrophysics Conference, AIP Conf. Proc., 1039, 214

Wang, H., Qiu, J., Jing, J., \& Zhang, H. 2003, ApJ, 593, 564

Wild, J. P., Smerd, S. F., \& Weiss, A. A. 1963, ARA\&A, 1, 291

Zhang, J., Dere, K. P., Howard, R. A., Kundu, M. R., \& White, S. M. 2001, ApJ, 559,452 